\title{
B Model Abstraction Combining Syntactic and Semantic Methods
}

\author{
Jacques Julliand $^{1}$, Nicolas Stouls ${ }^{2}$, Pierre-Christope Bué ${ }^{1}$, \\ and Pierre-Alain Masson ${ }^{1}$ \\ ${ }^{1}$ LIFC, Université de Franche-Comté \\ 16, route de Gray F-25030 Besançon Cedex \\ \{bue, julliand, masson\}@lifc.univ-fcomte.fr \\ ${ }^{2}$ Université de Lyon, INRIA \\ INSA-Lyon, CITI, F-69621, Villeurbanne, France \\ nicolas.stouls@insa-lyon.fr
}

\begin{abstract}
In a model-based testing approach as well as for the verification of properties by model-checking, B models provide an interesting solution. But for industrial applications, the size of their state space often makes them hard to handle. To reduce the amount of states, an abstraction function can be used, often combining state variable elimination and domain abstractions of the remaining variables. This paper illustrates a computer aided abstraction process that combines syntactic and semantic abstraction functions. The first function syntactically transforms a B event system $\mathrm{M}$ into an abstract one $\mathrm{A}$, and the second one transforms a B event system into a Symbolic Labelled Transition System (SLTS). The syntactic transformation suppresses some variables in $\mathrm{M}$. This function is correct in the sense that $A$ is refined by $M$. A process that combines the syntactic and semantic abstractions has been experimented. It significantly reduces the time cost of semantic abstraction computation. This abstraction process allows for verifying safety properties by modelchecking or for generating abstract tests. These tests are generated by a coverage criteria such as all states or all transitions of an SLTS.
\end{abstract}

Keywords: Model Abstraction, Syntactic Abstraction, Refinement.

The full version of this short paper is available as a research report:

\section{Reference}

[JSBM09] Julliand, J., Stouls, N., Bué, P.-C., Masson, P.-A.: B model abstraction combining syntactic and semantics methods. Research Report RR2009-04, LIFC - Laboratoire d'Informatique de l'Université de Franche Comté, 15 pages (November 2009) 